\title{
Protokoll der ordentlichen Ärztekammersitzung
}

\author{
Donnerstag, 19. Mai 2005, 9.30-17.00 Uhr, Kongresszentrum bea bern congress, Bern
}

Annamaria Müller Imboden, Generalsekretärin FMH

Anmerkung der Protokollführerin: Aus Gründen der Lesbarkeit werden die Traktanden nach Massgabe der Numerierung und nicht in der Reihenfolge der Diskussion aufgeführt. Letztere ist jedoch mittels eckiger Klammern [] gekennzeichnet.

Beginn der Sitzung: 9.40 Uhr.

\section{Begrüssung, Mitteilungen, Bestellung des Büros}

Der FMH-Präsident, Jacques de Haller, begrüsst die Anwesenden, dankt für das Erscheinen und stellt die Beschlussfähigkeit der Versammlung fest. Sodann gibt er verschiedene organisatorische und administrative Einzelheiten zum Tagesablauf bekannt. Aufgrund diverser Unabkömmlichkeiten wird die Reihenfolge der Traktanden umgestellt. Jacques de Haller begrüsst im besonderen den neuen Leiter der Abteilung Kommunikation, Daniel Lüthi, und gibt ihm Gelegenheit, sich kurz vorzustellen und seine Gedanken zur Rolle und zum Stellenwert der Kommunikation innerhalb der FMH darzulegen. Anschliessend wird der in den Ruhestand getretene Pressesprecher, Reto Steiner, gewürdigt.

\section{Als Stimmenzähler werden ernannt}

Valentin Audétat, Armand Bottani, Giorgio Bugliani, Peter Buser, Robert Cougn, Jörg Fritschi, Jean-Pierre Grob, Niklaus Hasler, Reto Ludwig, Rolf Schlumpf, Jürg Schwegler.

\section{Jahresbericht 2004}

Der statutarische Jahresbericht der FMH, erschienen in der Form des Geschäftsberichtes als Beilage zur Schweizerischen Ärztezeitung Nr. 18 vom 4. Mai 2005, gibt zu keinen Bemerkungen Anlass.

Antrag Nr. 2/1 (Zentralvorstand)

welcher die Genehmigung des Jahresberichtes beinhaltet, wird einstimmig ohne Gegenstimmen oder Enthaltungen angenommen.

\section{Jahresrechnung 2004 der FMH}

Die Generalsekretärin, Annamaria Müller Imboden, präsentiert die wichtigsten Ergebnisse des vergangenen Rechnungsjahres. Es schliesst wieder mit einem markanten Einnahmenüberschuss ab, der im wesentlichen aus ausserordentlichen Einnahmen eines gewonnenen Rechtsstreits, einer besseren Ertragslage der Weiterbildung und verringerten Ausgaben bei der Dignitätserhebung beruht. Es ist vorgesehen, den die Weiterbildung betreffenden Teil der Einnahmenüberschüsse rückwirkend den Reserven der AWF zuzuweisen. Der Vertreter der Kontrollstelle, Heribert Riesterer, PWC, bestätigt die Korrektheit der Rechnungslegung und empfiehlt deren Annahme. Nachdem vereinzelte Verständnisfragen betreffend den Rechtsstreit, das Sponsoring und die Beteiligungen beantwortet sind, schreitet die Kammer zur Abstimmung.

\subsection{Genehmigung}

Antrag Nr. 3.1/1 (Zentralvorstand)

der die Jahresrechnung 2004 inklusive der Rechnungen des «Hilfsfonds» sowie des «Fonds zur Förderung der Weiterbildung von Schweizer Ärzten in Entwicklungsländern» beantragt, wird einstimmig ohne Enthaltungen oder Gegenstimmen angenommen. Die transparente Rechnungslegung wird verdankt.

Christoph Ramstein, VEDAG-Präsident, verdankt die Arbeiten des Zentralvorstandes und der Mitarbeitenden des Generalsekretariates und seiner Abteilungen und spricht im Namen der Anwesenden ein grosses Lob aus.

\subsection{Decharge-Erteilung an den Zentral- vorstand}

\section{Antrag Nr. 3.2/1 (Zentralvorstand)}

Die Ärztekammer folgt den Empfehlungen der Kontrollstelle und erteilt den Mitgliedern des Zentralvorstandes für das Geschäftsjahr 2004 einstimmig, ohne Gegenstimmen oder Enthaltungen, Decharge. 


\section{Budget 2006 der FMH, Festsetzung des Mitgliederbeitrages 2006}

Annamaria Müller Imboden präsentiert das Budget für das Jahr 2006. Sie weist erneut auf die Umstellung zur strategischen Budgetierung hin und die damit verbundenen Änderungen in bezug auf Planung, Dokumentierung und Beschlussfassung. Das Budget 2006 wird bei gleichbleibenden Mitgliederbeiträgen als ausgeglichenes Budget präsentiert. Strategische Optionen sind die Weiterführung der AWF, die Positionierung der Abteilung Daten, Demographie und Qualität (DDQ) sowie des Tarifwesens und der Ausbau der Kommunikation. Daneben soll die internationale Kooperation verstärkt werden und eine Fokussierung der nationalen Kontakte stattfinden. Auch bezüglich des Finanzmanagements und der Rechnungslegung sind Veränderungen geplant, die das Budget als solches jedoch nicht betreffen.

Bezüglich der gestellten Anträge wird folgendes bemerkt: ein Antrag der SVM wurde vorgängig der Sitzung kurzfristig zurückgezogen, figuriert jedoch noch in den Unterlagen, desgleichen der Gegenantrag des Zentralvorstandes, der ebenfalls hinfällig ist. Für HIN wird ein Verschiebeantrag gestellt, die Finanzierung von HIN-Dienstleistungen soll über den Sommer erarbeitet und an der ausserordentlichen Ärztekammer im Dezember beschlossen werden. Die beiden übrigen Sonderbeitragsanträge werden insofern diskutiert, als die Kammerdelegierten inskünftig deren Integration in die Grundbeiträge wünschen. Der Zentralvorstand begrüsst diesen Wunsch und nimmt ihn für das Budget 2007 entsprechend entgegen.

\section{Antrag Nr. 4/1 (Zentralvorstand)}

auf Genehmigung des vorgelegten Budgets für das Jahr 2006 wird mit 1 Gegenstimme und ohne Enthaltungen mit den nachfolgenden Mitgliederbeiträgen angenommen:

Mitgliederbeiträge 2006

\begin{tabular}{llll} 
Beitragskategorie 1 & Selbständig tätige Ärztinnen und Ärzte & Fr. 530.- \\
\hline Beitragskategorie 2 & $\begin{array}{l}\text { Unselbständig tätige Ärztinnen und Ärzte in leitender } \\
\text { Funktion }\end{array}$ & Fr. 530.- \\
\hline Beitragskategorie 3 & $\begin{array}{l}\text { Unselbständig tätige Ärztinnen und Ärzte nicht in leitender } \\
\text { Funktion und nicht in FMH-Weiterbildung }\end{array}$ & Fr. 265.- \\
\hline Beitragskategorie 4 & Ärztinnen und Ärzte in Weiterbildung & Fr. 265.- \\
\hline Beitragskategorie 5 & $\begin{array}{l}\text { Ärztinnen und Ärzte mit Wohnsitz und Berufstätigkeit } \\
\text { im Ausland }\end{array}$ & Fr. 132.- \\
\hline Beitragskategorie 6 & Vorübergehend nicht als Ärztin/Arzt berufstätige Mitglieder & Fr. 132.-
\end{tabular}

\section{Antrag Nr. 4/2 (Marc Müller, KHM)}

beantragt die nochmalige Weiterführung der finanziellen Unterstützung der Praxisassistenz von FMH-Mitgliedern in Weiterbildung für drei Jahre in der Form eines Sonderbeitrags von Fr. 25.- pro Mitglied, geltend für die Beitragskategorien 1, 2, 3 und 4. Der Sonderbeitrag unterliegt der jährlichen Budgetgenehmigung. Zur Begründung wird angeführt, dass die Weiterbildungsmöglichkeit in der Hausarztpraxis nach wie vor sehr geschätzt und von den Absolventen und Absolventinnen als unabdingbar betrachtet wird. Die Praxisassistenz stellt eine zentrale Massnahme dar, um dem Ärztenachwuchs einen lebensnahen Einblick in den Beruf zu gewähren. Es zeichnet sich ab, dass das Pilotprojekt in eine reguläre Form der Weiterbildung überführt werden kann, da zunehmend auch der politische Boden hierfür bereit ist. Unter diesen Gesichtspunkten wird der Antrag mit 1 Gegenstimme und 6 Enthaltungen angenommen.

\section{Antrag Nr. 4/3 (Zentralvorstand)}

Auch der Sonderbeitrag für die Finanzierung der nationalen Datenkonsolidierung in der Höhe von Fr. 20.- pro Mitglied, geltend für die Beitragskategorien 1 und 2, wird für das Jahr 2006 erneut beantragt. Die Bereitstellung von Mitteln für die Sammlung und Aufbereitung von Daten ist unumstritten, die Kammer stellt jedoch die Frage, warum keine Integration der Finanzierung in die ordentlichen Mittel der FMH erfolgt. Der Zentralvorstand antwortet, dass dies erfolgen wird, sobald der Initialaufwand abgeschlossen ist, für welchen indessen besondere Mittel benötigt werden. Die Kammer kann der Logik folgen und genehmigt den Beitrag mit 2 Gegenstimmen und 5 Enthaltungen.

\section{Antrag Nr. 4/4 (Zentralvorstand)}

ist ein «exotischer» Antrag, da er wünscht, über die Finanzierung von HIN-Dienstleistungen für alle FMH-Mitglieder erst an der ausserordentlichen Ärztekammersitzung vom 2. Dezember 2005 zu befinden. Nach einer kurzen Erläuterung seitens des Zentralvorstandes über die Unmöglichkeit, der Kammer in der vorgegebenen Frist ein konkretes Projekt inklusive eines seriösen Finanzierungsvorschlags zu unterbreiten, stimmen die Delegierten dem Antrag ohne Gegenstimme mit 5 Enthaltungen $z u$. An den Zentralvorstand wird dabei die Erwartung herangetragen, Ende Jahr nicht bloss mit einem (neuerlichen) Verlängerungsantrag aufzuwarten. 


\section{Aus-, Weiter- und Fortbildung AWF}

Der Ressortleiter «Medical Education», Max Giger, gibt einen kurzen Überblick über den aktuellen Stand sowie die Entwicklungen im Bereich der Weiter- und Fortbildung. Kernpunkte sind die Akkreditierung der FMH, die Schaffung von eidgenössischen Facharzttiteln (FAT) sowie die Arbeiten am Medizinalberufegesetz (MedBG). Das zuständige Departement ist nicht immer gleicher Meinung wie die FMH, es besteht jedoch Zuversicht auf konstruktive Lösungen in den verschiedenen Belangen.

\subsection{Schaffung eines Facharzttitels Handchirurgie}

\section{Antrag Nr. 5.1/1 (Zentralvorstand)}

Der Ärztekammer wird die Schaffung eines FAT Handchirurgie beantragt. Damit sollen die Bemühungen für eine konkurrenzfähige, qualitativ und quantitativ hochstehende Handchirurgie unterstützt werden. Die KWFB befürwortet die Schaffung des FAT, hingegen nicht sämtliche der betroffenen Fachgesellschaften. Die Kritik richtet sich in erster Linie an die mangelhafte EUKompatibilität, an mögliche Doppelspurigkeiten in den Weiterbildungsprogrammen sowie Probleme im Zusammenhang mit der Besitzstandswahrung (Dignität). Daneben wird die Frage aufgeworfen, was geschieht, wenn der zuständige Departementsvorsteher die Schaffung des FAT ablehnt. Der Zentralvorstand plädiert dafür, tarifarische Fragen nicht vor diejenigen der Qualität zu stellen. Die Zuständigkeit zur Schaffung eidgenössischer Titel liege klar beim Bund, man werde nicht zweigleisig fahren (keine parallelen «FMH-FAT» zu den eidg. FAT). In Würdigung all dieser Gesichtspunkte nimmt die Kammer den Antrag mit 9 Gegenstimmen und 19 Enthaltungen an.

\subsection{Kommission für Weiter- und Fortbildung KWFB}

\section{a) Einsitznahme des Schweizerischen Vereins Homöopathischer Ärztinnen und Ärzte (SVHA)}

\section{Antrag Nr. 5.2.a/1 (Bruno Ferroni, SVHA)}

beantragt die Einsitznahme des Schweizerischen Vereins Homöopathischer Ärztinnen und Ärzte (SVHA) in die Kommission für Weiter- und Fortbildung (KWFB). Die Voraussetzungen hierfür sind gegeben, da gemäss Art. 41 Abs. 4 der FMHStatuten Ärztevereinigungen, welche für einen Schwerpunkt oder einen Fähigkeitsausweis ver- antwortlich zeichnen, in der KWFB Einsitz nehmen können. Der Antrag wird mit 76 Stimmen bei 47 Gegenstimmen und 21 Enthaltungen angenommen.

\section{b) Einsitznahme der Schweizerischen Ärztegesellschaft für Neuraltherapie (Regulationstherapie) nach Huneke (SANTH)}

Antrag Nr. 5.2.b/1 (Andreas Beck, SANTH) steht in Analogie zum Antrag 5.2.a/1 und verlangt die Einsitznahme der Schweizerischen Ärztegesellschaft für Neuraltherapie (Regulationstherapie) nach Huneke (SANTH) in die Kommission für Weiter- und Fortbildung (KWFB). Auch dieser Antrag wird mit 76 Stimmen bei 47 Gegenstimmen und 28 Enthaltungen angenommen.

\section{Politische Aktivitäten}

Der Vizepräsident der FMH, Nationalrat Yves Guisan, gibt einen Überblick über die FMH-relevanten parlamentarischen Beratungen. Es handelt sich konkret um folgende Punkte:

- Das Vernehmlassungsverfahren über den Zulassungsstopp (Verordnung vom 3. Juli 2002 über die Einschränkung der Zulassung von Leistungserbringern zur Tätigkeit zu Lasten der obligatorischen Krankenpflegeversicherung): Zum Zulassungsstopp hat sich die FMH vernehmen lassen, ihre grundsätzliche Missbilligung ausgedrückt, ihre Kritikpunkte eingebracht und an die Verantwortung des Bundes bezüglich möglicher Auswirkungen auf die medizinische Versorgung appelliert.

- Das MedBG: Bei der Beratung zum MedBG wurde mit Freude zur Kenntnis genommen, dass für die Weiterbildung nur eine Organisation zuständig sein soll. Erfreulich ist auch die Solidarität mit anderen Berufsorganisationen und den Universitäten. Weniger erfreulich die Ablehnung des «Weiterbildungsanspruchs». Ein zusätzlicher, noch wenig erkannter, sich aber zuspitzender Problemkreis droht die obligatorische Berufshaftpflichtversicherung zu werden, da es den Ärztinnen und Ärzten zunehmend schwerfällt, finanziell tragbare Versicherungspolicen abzuschliessen. Viele Haftpflichtversicherer deklarieren die Ärzteschaft als Hochrisikogruppe mit entsprechenden vertraglichen Konsequenzen.

- Die Revision des KVG und ihre Stossrichtung: Die Ständeratskommission wird sich mit Fragen zur Spitalfinanzierung und der Vertragsfreiheit befassen. 
Allgemein ist man daran, die Lobbyingarbeit der FMH im Parlament zu verstärken und zu professionalisieren.

In der anschliessenden Diskussion wird der Einsatz der FMH bestens verdankt und gleichzeitig bedauert, dass den Anliegen der Ärzteschaft anscheinend wenig Rechnung getragen wird. Die Kantonalgesellschaften werden geheissen, Engpässe in der Versorgung genau zu dokumentieren und sie den zuständigen Kantonsbehörden zur Kenntnis zu bringen. Schliesslich sind es die Kantone, welchen die Umsetzung des Zulassungsstopps obliegt.

\section{Antrag Nr. 6/1 (Hans Kurt, FMPP)}

der den Zentralvorstand darum bittet, alles zu unternehmen, damit der Zulassungsstopp aufgehoben wird oder zumindest das Problem der Teilzeitarbeit und des Unterbruches der ärztlichen Tätigkeit präzisiert und gelöst werden kann, wird im Sinne einer Aufforderung an den Zentralvorstand überwiesen und von diesem bereitwillig entgegengenommen.

\section{Tarife und Verträge}

Der Leiter des Ressorts und der ZV-«Task-Force» Tarife, Franco Muggli, gibt verschiedene Informationen rund um den TARMED ab, nicht ohne vorher allen Mitwirkenden herzlich zu danken. In den Arbeiten und Verhandlungen hat sich gezeigt, wie wichtig es ist, über eigene Daten zu verfügen. Dies auch und nicht zuletzt, weil der ursprünglich «betriebswirtschaftliche» Tarif nun in einen «volkswirtschaftlichen» Tarif überführt worden ist, sprich, bedrohlich in die Nähe eines ambulanten Globalbudgets gerückt ist. Offen und ungeklärt sind nach wie vor Fragen über die Parameter und Messgrössen der WZW-Kriterien. Nichtsdestotrotz ist der politische Druck auf eine Kostenkontrolle via Tarifmechanik ungebrochen. Mit einleuchtenden Beispielen zeigt Franco Muggli auf, dass «Daten haben» allein noch nicht ausreicht, sondern die Ergebnisse mit grosser Sorgfalt interpretiert werden müssen und bei näherer Betrachtung ein immer differenzierteres Bild abgeben. Derzeit ist man daran, mit dem Projekt «NAKO» die Datenbewirtschaftung aufzubauen und sicherzustellen. Dies ist um so wichtiger, da die Erarbeitung und der Abschluss der sogenannten «TARMED-Nachfolgeverträge» auf Hochtouren laufen. Anschliessend weist er auf das komplexe und langwierige Verhandlungs- und Beschlussprozedere in den Tarifgremien hin und plädiert für Verständnis, wenn die Anträge der Ärztegesellschaften teilweise erheb- lich zeitliche Verzögerungen und inhaltliche Verwässerungen erfahren. Tarifverhandlungen sind eben keine «Lieferaufträge».

Der scheidende Präsident der G7, Res Haefeli, präsentiert den Stand der Dinge bei der Nachfolgeregelung der Kostenneutralität. Einen Monat vor Ende der Kostenneutralitätsphase bzw. vor der Festlegung der definitiven Taxpunktwerte geht es hektisch zu und her. Diverse Punkte sind noch zu klären und zu bereinigen, unter anderem markante Ungereimtheiten in der Datenbasis von santésuisse. Ein heikler Punkt ist die Handhabung des Kompensationsvolumens, welches sich nach den «zu viel» bezogenen oder «nicht ausgeschöpften» Volumina der Kantone bzw. der Taxpunktwertregionen bemisst und sich in Differenz zum retrospektiv festgestellten, kostenneutralen Taxpunktwert ermittelt. Einige Kantone haben massive Rückzahlungspflichten, andere hätten noch etwas «zugut». Anhand der Korrekturvolumina werden ebenfalls die interimistischen und die definitiven Taxpunktwerte bestimmt. In einer an die Ärztekammer anschliessenden Konferenz der Kantonalpräsidenten soll diese Thematik vertieft diskutiert und das konkrete Vorgehen beschlossen werden. Dabei geht es insbesondere um die brisante Frage der Taxpunktwertharmonisierung. Sie ist auch in der an die Ausführungen von Res Haefeli anschliessenden Diskussion ein heiss umstrittenes Thema. In der Sache zwar unbestritten, scheiden sich die Geister über den Weg und die Marschgeschwindigkeit. Ebenfalls moniert wird das Ausblenden des spitalambulanten Bereichs. Hier kann jedoch FMH-seitig nichts unternommen werden, da die Verhandlungskompetenz in den Händen des Spitalverbandes liegt. Schliesslich informiert Res Haefeli noch über die geplante Leistungs- und Kostenvereinbarung, welche an die Stelle der Kostenneutralitätsvereinbarung treten soll. Sie beinhaltet - im Gegensatz zu letzterer - jedoch keine verbindlichen Vorgaben, sondern lediglich Empfehlungen an die kantonalen Ärztegesellschaften, denen nach Beendigung der Kostenneutralitätsphase die Verhandlungsführung wieder zukommt. Ziel ist es, möglichst einheitliche und partnerschaftliche Regelungen für die Zielfindung, d.h. die Ermittlung der Taxpunktwerte, zu schaffen und den Gang über die Beschwerdeinstanzen $\mathrm{zu}$ vermeiden. Nicht wegbedungen werden kann der Einfluss des Preisüberwachers, der von der Kantonsregierung im Genehmigungs- und/oder Festsetzungsverfahren zwingend angehört werden muss. Ebenfalls problematisch gestaltet sich die angestrebte Konvergenz, wenn die Vertragsräume ausschliesslich kantonal und getrennt nach 
freier Praxis und Spitalambulatorium gehandhabt werden. Aber so ist nun einmal die gesetzliche Übungsanlage. Nach erfolgter Diskussion wird der Einsatz von Res Haefeli ganz herzlich verdankt.

Franco Muggli schildert kurz die Situation im UVG-Bereich. Hier ist die Fallkostenstabilitätsphase schon seit längerer Zeit abgeschlossen und der definitive Taxpunktwert festgelegt. Mit Schützenhilfe des BAG macht die MTK nun Druck auf den Abschluss einer Vereinbarung, die eine erneute und schliesslich permanente Kostensteuerung beinhaltet. Tatsache ist indessen, dass gültige, ungekündigte Verträge existieren und der Bund, rechtlich gesehen, über keine Entscheidungsmacht verfügt.

Olivier Kappeler, mit Dignitätsfragen beauftragtes Zentralvorstandsmitglied, berichtet über den Stand der Dignitätsdatenbank, die in der zweiten Jahreshälfte nun anscheinend doch noch in Betrieb genommen werden soll. Der Abbau der Pendenzen schreitet planmässig voran und sollte bis dahin abgeschlossen sein, ein konstruktives und speditives Mitwirken der betroffenen Ärztinnen und Ärzte vorausgesetzt. Parallel dazu hat die FMH mit Zustimmung von santésuisse bei der Wettbewerbskommission eine Anfrage deponiert. Diese zielt auf zwei Fragestellungen ab: Ist das Kartellrecht im Bereich der Dignität anwendbar? Ersetzt die bundesrechtliche Genehmigung allenfalls fehlende gesetzliche Grundlagen? Der Eingang wurde bestätigt, eine Antwort darauf steht noch aus.

\section{Antrag Nr. 7/1 (Jürg Schlup, Bern)}

beinhaltet eine Anfrage an den Zentralvorstand, was dieser in der Angelegenheit des per Ende Jahr auslaufenden Tarifvertrags für paramedizinische Leistungen in der Arztpraxis zu tun gedenkt bzw. was den Mitgliedern zu tun empfohlen wird. Die Rechtslage erscheint alles andere als klar. Der stellvertretende Generalsekretär der FMH, Hanspeter Kuhn, führt aus, dass es bereits einschlägige Bundesgerichtsentscheide zur Thematik «angestelltes Personal in der Arztpraxis» gibt. Der Zentralvorstand ist zuversichtlich, mit santésuisse in Bälde eine gemeinsame Lösung zu finden. Mit dieser Auskunft gibt sich der Antragsteller zufrieden.

\section{Antrag Nr. 7/2 (Charles Selleger, Genf)}

verlangt vom Zentralvorstand, in den Verhandlungen mit den Versicherern im System des «tiers payant» (KVG und UVG) zu erreichen, dass die Zahlungen an die Ärzte mittels ESR erfolgen, wenn der Arzt für die Ausstellung seiner Rechnungen ebenfalls den ESR-Einzahlungsschein (oranger Einzahlungsschein mit Referenznummer) verwendet. Nachdem die Informatisierung der Arztpraxen obligatorisch geworden ist, sei es nicht nachvollziehbar, dass sich die Versicherer nicht dem ESR-System anschliessen, welches zu einer Vereinfachung der elektronischen Datenverwaltung in der Arztpraxis führt. Der Antrag wird ohne Abstimmung vom Zentralvorstand als Verhandlungsauftrag entgegengenommen.

\section{Antrag Nr. 7/3 (Beat Manser, Luzern)}

wünscht die Beauftragung der G7, über eine national symmetrische Verteilung des Kompensationsvolumens zu verhandeln. Es sei stossend, dass diejenigen Kantone, welche zu Beginn ein sehr niedriges Kostenniveau auswiesen, das während der Kostenneutralitätsphase zwar leicht gestiegen wäre, aber immer noch unter dem schweizerischen Durchschnitt läge, dafür mit tiefen Taxpunktwerten «bestraft» würden. Der Antrag wird aus formellen Gründen zurückgezogen: Die Ärztekammer ist nicht legitimiert, den Kantonalgesellschaften Verhandlungsaufträge zu erteilen. Der Antrag wird jedoch im gestellten Sinne an die gleichentags stattfindende Konferenz der Kantonalpräsidenten überwiesen und soll dort diskutiert werden.

\section{Standesordnung}

[behandelt nach Traktandum 11]

\subsection{Aufnahme von Richtlinien der SAMW}

Bisher gab es nur eine einzige Richtlinie für den Umgang mit Sterbenden. Da dies, so berichtet Ursula Steiner-König, Vizepräsidentin der FMH, den Gegebenheiten nicht hinreichend Rechnung trug, wurde diese ausdifferenziert und vier Richtlinien für verschiedene Kontexte erarbeitet. Die Schulung der betroffenen Ärztinnen und Ärzte ist zwar nicht explizit aufgeführt, jedoch implizit in den Richtlinien erhalten.

\section{Antrag Nr. 8.1/1 (Zentralvorstand)}

schlägt vor, die vier neuen medizinisch-ethischen Richtlinien

- Grenzfragen Intensivmedizin;

- Behandlung von zerebral schwerstgeschädigten Langzeitpatienten;

- Behandlung und Betreuung von älteren, pflegebedürftigen Betreuungen;

- Betreuung von Patienten am Lebensende

der Schweizerischen Akademie der Medizinischen Wissenschaften SAMW zur Betreuung von Patientinnen und Patienten am Lebensende in 
die Standesordnung FMH aufzunehmen. Er wird mehrheitlich bei 1 Gegenstimme und 4 Enthaltungen angenommen.

\section{Projekt HOP-Line}

[behandelt nach Traktandum 7]

Gäste zu diesem Traktandum: Dr. Christiane Margairaz, Interdisziplinäre Beratung für Medizin und Gewaltprävention (CIMPV), Universitätsspital Genf, und Dr. Judit Pòk, Leitende Ärztin an der Frauenpoliklinik des Universitätsspitals Zürich.

Die Vizepräsidentin der FMH, Ursula SteinerKönig, führt in das Traktandum ein und beginnt mit einer Rekapitulation des Beschlusses der Ärztekammer vom 11. Oktober 2003, die den Zentralvorstand beauftragte, sich mit dem Thema «Professional Sexual Misconduct» (PSM) zu befassen und der ordentlichen Ärztekammer 2005 ein konkretes Projekt zu unterbreiten. Der Zentralvorstand setzte in der Folge für die Umsetzung des Auftrags eine Arbeitsgruppe unter der Leitung von Werner Tschan ein. Diese Gruppe legte im Herbst 2004 ihren Bericht vor und schloss ihn mit 17 Empfehlungen an die Ärztekammer ab. Im Vorfeld zur heutigen Ärztekammersitzung kam es in den Medien zu diversen, teilweise reisserischen Berichterstattungen im Zusammenhang mit PSM unter Hinweis auf dessen Thematisierung an der heutigen Kammer. Im Namen des Zentralvorstandes macht Ursula Steiner-König hierzu folgende Bemerkungen: Das neue MedBG sieht vor, dass die zuständige kantonale Aufsichtsbehörde Disziplinarmassnahmen anordnen kann. Schon jetzt haben die Standeskommissionen der Ärzteschaft Sanktionsmöglichkeiten, die bis zur Veröffentlichung in den Publikationsorganen der Ärztegesellschaften inklusive der FMH, inklusive einer Mitteilung an die zuständige Behörde und die Versicherer gehen. Da die Schweiz - im Unterschied etwa zu den USA - keine Meldepflicht von PSM-fälligen Ärztinnen und Ärzten kennt, ist die in den Medien kolportierte Zahl von hochgerechnet jährlich 14000 Übergriffsfällen weder glaubhaft noch nachgewiesen. Das mediale Vorpreschen ist bedauerlich und wirft ein ungünstiges Schlaglicht auf ein sensibles und schmerzhaftes Thema.

Christiane Margairaz zeigt in ihrer Präsentation das Deletäre am Abusus von Ärzten und Ärztinnen gegenüber Patienten und Patientinnen auf: Als fatale Konsequenz werde der kranke Mensch dadurch noch kränker, was einerseits dem in der Standesordnung hinterlegten Heilungspostulat widerspricht und andererseits das
Vertrauensverhältnis zerstört, die Grundbedingung ärztlichen Handelns schlechthin. Zudem gerät die gesamte Ärzteschaft in Verruf. Ziel muss deshalb sein, bereits in der Ausbildung die angehenden Ärztinnen und Ärzte darauf hinzuweisen, dass die «Machtposition» des Arztes oder der Ärztin niemals zur Erfüllung eigener Bedürfnisse benützt werden darf und dass Sexualität und Erotik in der Arzt-Patienten-Beziehung nichts zu suchen haben.

Judit Pòk fasst den Inhalt des Berichts der Arbeitsgruppe zusammen. Er fokussiert sich auf PSM, gilt aber grundsätzlich für alle Formen des beruflichen Fehlverhaltens. Im weiteren zielt er klar auf ein Hilfsangebot für Ärztinnen und Ärzte $\mathrm{ab}$ - darum auch der Name «HOP» Help Our Physicians - wissend, dass dadurch auch implizit den Patientinnen und Patienten geholfen wird. Kernstück des Berichts und der darin enthaltenen Empfehlungen ist die individuelle Beratung. Die letzte der Empfehlungen expliziert das vorgeschlagene Beratungsangebot im Sinne einer Telefonlinie, einer Website und eines Pools von Fachleuten, die für die Beratungstätigkeit zur Verfügung stünden. Letzterer wäre noch zu gründen und die Personen zu schulen. Judit Pòk weist allerdings darauf hin, dass die Arbeitsgruppe nicht zwingend die Einhaltung der Empfehlungen fordert, sondern ihr Kernanliegen darin sieht, dass sich die FMH mit der Thematik ernsthaft befasst, sich in aller Deutlichkeit nach innen und nach aussen von PSM distanziert und konkrete Massnahmen zur Prävention und Hilfestellung ergreift.

Der Bericht der Arbeitsgruppe sowie die enthaltenen Empfehlungen werden in der anschliessenden Diskussion unterschiedlich gewürdigt, insbesondere kann die quantitative und qualitative Dramatik nicht nachvollzogen werden. Absolut einig sind sich alle Redner, dass bei PSM «zero tolerance» gelten soll und das Thema ernstzunehmen ist. Als problematisch im Vorschlag der Arbeitsgruppe zur Telefonlinie wird erachtet, dass nicht abgeklärt worden ist, ob sich die Betreiber nicht straffällig machen, wenn sie Kenntnis von Übergriffen erhalten und diese den zuständigen Behörden melden. Immerhin ist sexueller Missbrauch ein ahndbarer Straftatbestand. Des weiteren wird die Frage aufgeworfen, ob es vertretbar ist, während mehrerer Jahre namhafte Geldbeträge in einen Testbetrieb zu stecken, wenn keine Abklärungen darüber getroffen wurden, ob und wieviele Telefonanrufe oder E-Mail-Kontakte PSM-betroffener Kolleginnen und Kollegen pro Jahr zu erwarten sind. Bei der Beratungsstelle in Basel hat sich bisher noch kein einziger Täter gemeldet. 


\section{Antrag Nr. 9/1 (Zentralvorstand)}

wird durch René Raggenbass, Co-Leiter des Ressorts «Gesundheit und Prävention», vorgestellt. René Raggenbass hält fest, dass der Zentralvorstand PSM vollumfänglich verurteilt. Der Zentralvorstand ist jedoch der Meinung, dass die Vorschläge der Arbeitsgruppe HOP-Line keine geeigneten Massnahmen zur Lösung des Problems darstellen (Telefonlinie ins Generalsekretariat? Website?) und dass ihr Bezug zur Realität in der Schweiz nicht überprüft wurde. Aus diesem Grund schlägt der Zentralvorstand der Ärztekammer vor, das Projekt an die Arbeitsgruppe zur Überarbeitung zurückzuweisen, mit dem Auftrag, ein Pilotprojekt zu konzipieren, das auf drei Jahre befristet ist und mit einem jährlichen Budget in der Grössenordnung von Fr. 10 000.- realisiert werden kann. Ziel des Pilotprojekts soll sein, konkrete, realitätsnahe und ausgetestete Vorschläge für Massnahmen gegen PSM zu vorzulegen.

\section{Eintretensvotum}

Zunächst einmal stimmt die Kammer darüber ab, ob das Thema PSM überhaupt weiterverfolgt werden soll. Die Delegierten sprechen sich bei 3 Gegenstimmen und 8 Enthaltungen deutlich dafür aus.

Bei der anschliessenden Abstimmung HOPLine- gegen ZV-Antrag unterliegt ersterer mit 0 Stimmen bei 7 Enthaltungen. Der Antrag des Zentralvorstandes ist somit klar angenommen.

\section{Einheitliche gesamtschweizerische Notrufnummer}

[behandelt nach Traktandum 8]

Marc Müller, Präsident der Plattform Rettungswesen FMH, informiert über die Beratungen und Empfehlungen der Plattform zur gesamtschweizerisch einheitlichen (dreistelligen) Notrufnummer: Bereits 1996 hat die FMH in ihren 12 Thesen zum Rettungswesen in der Schweiz (in der «Schweizerischen Ärztezeitung» publiziert [1]) unter These 2 festgehalten, dass die Sanitätsnotrufnummer 144 als universelle Anlaufstelle für alle medizinischen Notfallsituationen in der ganzen Schweiz flächendeckend und gebührenfrei einzurichten sei. Die Plattform erachtet dieses Postulat auch heute noch als adäquat und empfiehlt aus diesem Grund, auf die Einführung einer eigenen dreistelligen Nummer für den ärztlichen Notfalldienst zu verzichten, da diese nur zu Verwirrungen führen würde. Derzeit ist die Plattform daran, Entscheidungsgrundlagen zu Handen der kantonalen Ärztegesellschaften, der Kantone - in deren Kompetenz die Organisation des Notfalldienstes liegt - sowie der GDK zu erarbeiten, welche eine Übersicht der verschiedenen Organisationsformen des ärztlichen Notfalldienstes in der Schweiz beinhalten. Der IVR hat seine Mitwirkung zugesichert.

\section{Qualitätsverständnis der Schweizerischen Ärzteschaft}

[behandelt nach Traktandum 9]

Der VEDAG-Co-Präsident, Christoph Ramstein, führt in einer kurzen Einleitung den Hintergrund des zur Diskussion stehenden Antrags aus. VEDAG-Mitglied Hans-Anton Vogel präsentiert das dem Antrag zugrundeliegende «Leitbild Qualität der Schweizer Ärzteschaft», welches den Präsidentinnen und Präsidenten der in der Ärztekammer vertretenen Organisationen am 21. April 2005 präsentiert und in der «Schweizerischen Ärztezeitung» publiziert wurde [2]. Den Initianten ist wichtig festzuhalten, dass es keine VEDAG-interne Angelegenheit ist, dass die vorgeschlagene Arbeitsgruppe offen ist und sich aus Vertreterinnen und Vertretern der ganzen Schweiz zusammensetzen soll und das Fachwissen in der Form von Experten des Generalsekretariates integriert wird.

Antrag Nr. 11/1 (Christoph Ramstein, VEDAG) Der VEDAG beantragt die Gründung einer «Arbeitsgruppe Qualität FMH», welche den Auftrag hat,

a. das Leitbild laufend weiterzuentwickeln;

b. die Umsetzung der Inhalte des Leitbildes zu fördern;

c. ein Konzept für eine Qualitätsplattform sowie für die Ausbildung der Qualitätsbeauftragten und der Qualitätsaudits zu erarbeiten;

d. auf der Grundlage des Qualitätsverständnisses der Schweizer Ärzteschaft mit den Ansprechpartnern in Verhandlungen zu treten.

Der Antrag wird mit 5 Gegenstimmen bei 9 Enthaltungen angenommen.

\section{HIN: Zusammenarbeit, Projekte}

Verschoben auf die Ärztekammer vom 2. Dezember 2005 .

\section{Varia}

Keine Meldungen.

Der FMH-Präsident schliesst die Sitzung um 16.35 Uhr. 